\title{
First principles study of liquid uranium at temperatures up to $2050 \mathrm{~K}$.
}

\author{
Beatriz G del Rio, Luis E González and David J González \\ Departamento de Física Teórica, Universidad de Valladolid, Valladolid, SPAIN \\ E-mail: luisen@metodos.fam.cie.uva.es
}

\begin{abstract}
Uranium compounds are used as fissile materials in nuclear reactors. In present day reactors the most used nuclear fuel is uranium dioxide, but in generation-IV reactors other compounds are also being considered, such as uranium carbide and uranium mononitride. Upon possible accidents where the coolant would not circulate or be lost the core of the reactor would reach very high temperatures, and therefore it is essential to understand the behaviour of the nuclear fuel under such conditions for proper risk assessment. We consider here molten metallic uranium at several temperatures ranging from 1455 to 2050 K. Even though metallic uranium is not a candidate for nuclear fuel it could nevertheless be produced due to the thermochemical instability of uranium nitride at high temperatures. We use first principles techniques to analyse the behaviour of this system and obtain basic structural and dynamic properties, as well as some thermodynamic and transport properties, including atomic diffusion and viscosity.
\end{abstract}

PACS numbers: 61.20.Ja, 61.20.Lc, 61.25.Mv, 62.50.+p, 62.60+v, 71.15.Pd

Keywords: liquid U, ab initio simulations.

Submitted to: J. Phys.: Condens. Matter 


\section{Introduction.}

Solid uranium dioxide is well known to be the most used fuel in nuclear power plants throughout the world. The knowledge of its properties is therefore of utmost importance, and a vast literature has been dedicated to their study (see for instance $[1,2,3]$ and references therein).

Issues of present day nuclear reactors such as the generation of long-lived high activity radioactive waste of difficult disposal, their possible use for production of material for nuclear weapons, and the idea of increasing the security upon possible accidents, led to the creation of the generation-IV international forum [4] in order to address the best routes for future development of nuclear reactors. At present there are several favoured designs for generation-IV reactors, namely, sodium cooled fast reactors, lead (or leadbismuth) cooled fast reactors, gas cooled fast reactors, molten salt reactors, very high temperature reactors and supercritical water cooled reactors. Additionally to designs that improve security and efficiency, a most important aspect in generation-IV reactors is the nuclear fuel, with special emphasis in those fuels that enable efficient reprocessing or breeding of new fuel during operation. So, in addition to uranium dioxide and mixed uranium plutonium dioxide, other candidate fuels are being considered such as uranium carbide, uranium mononitride, metallic uranium alloyed with zirconium or molybdenum, and thorium based fuels. In the particular case of the nitride (UN) there are some advantages as compared to uranium dioxide: it has a higher thermal conductivity, so that fuel elements would suffer lower temperature gradients, it has a higher density of fissile material, it is easily adaptable to current reprocessing technologies, and moreover it has good compatibility with $\mathrm{He}, \mathrm{Na}$ and $\mathrm{Pb}$ as coolants. However it has an important drawback: if it is not in an atmosphere with sufficient partial pressure of $\mathrm{N}_{2}$, UN is thermochemically unstable at high temperatures and decomposes into gaseous $\mathrm{N}_{2}$ and liquid $\mathrm{U}$ [5]. In fact at very high temperatures liquid $\mathrm{U}$ can also evaporate due to the increased vapor pressure as the temperature increases.

Consequently, liquid U (l-U) may appear, even if unwanted, in any situation where UN fuel is subject to high temperatures, be it during its production, where high temperature sintering may be used to manufacture the fuel pellets, or upon normal operation in the reactor, or more importantly upon possible accidents where the coolant would be lost or would not circulate for whatever reason. Consequently, understanding the properties and behaviour of $\mathrm{l}-\mathrm{U}$ is essential for assessing the risks and devising the most adequate procedures to follow if such events ever happen.

There is a plethora of information about the properties of solid uranium, at ambient and also at higher pressures, as obtained from experiments and from theoretical calculations. However the properties of the liquid have scarcely been experimentally or theoretically addressed. Even though the melting temperature is not extreme $(1405 \mathrm{~K})$ there have been, to our knowledge, no diffraction measurements of its structure, and we are aware of only very few (and most of them, old) measurements of basic thermophysical properties [3], like the density or the viscosity.

The properties of solid U are very peculiar among single-component metals, showing several unique characteristics [6]. The ultimate reason for these peculiarities is the contribution of the $5 f$ electrons to the bonding properties of the system, a question that adds scientific interest to the technological one. Solid U shows a unique charge density wave as ground state at ambient pressure, that is not associated to a spin density wave. At temperatures above $41 \mathrm{~K}$ it transforms into a unique orthorhombic structure, the $\alpha$ phase, with four atoms in the unit cell, which is not shared by any other element of the periodic table. It is highly anisotropic, and many studies have been devoted to the investigation of its properties (lattice constants, elastic constants, phonons, etc) as a function of both temperature and pressure. Experimental studies [7] have also been complemented by theoretical ones. Many different interatomic potentials have been constructed specifically to study the properties of the $\alpha$ phase, including popular types as the embedded atom method (EAM) [8] or modified embedded atom method (MEAM) [9], and more specific forms such as the charge optimized many body potential (COMB) [10], the so called long range potential (LR) [11] and also recently potentials constructed through machine learning [12].

The parameters of the potentials are usually fitted so as to reproduce experimental and/or more accurate theoretical calculations, such as those obtained using first principles techniques, which take into account all the bonding characteristics in a self-consistently 
way by considering the electronic degrees of freedom explicitly. Among these ab initio methods, density functional theory (DFT) [13] has become a most useful tool which is exploited in first principles molecular dynamics (FPMD) calculations, in which ionic motion is described by classical dynamics, by using a (multibody) potential energy given by the electronic ground state energy (obtained within DFT), following the Born-Oppenheimer approximation. First principles calculations for $\alpha$-U have indeed been performed using different forms of exchange and correlation, number of electrons considered and methods to describe their interaction with the ions/nuclei $[14,15,16]$. At this point it is interesting to mention the possible importance of spin-orbit (SO) coupling in the calculation of the properties of the system, given the heavy character of the $\mathrm{U}$ atom. Söderlind and coworkers [14] performed all electron first principles linear muffin tin calculations, including SO interaction, while many of the other ab initio studies did not include it $[15,16]$. The comparison between the two types of calculations showed a small effect on the equilibrium lattice constants. Moreover, calculations of the phonon spectra both including and ignoring SO effects produced identical results [16]. This showed that for a given ionic configuration the forces are not much influenced by SO effects.

At pressures below $3 \mathrm{GPa}$, and in a small pocket of temperatures around $1000 \mathrm{~K}$, a tetragonal $\beta$ phase is formed, with 30 atoms in the unit cell.

Finally, there is a high temperature $\gamma$ phase from which U melts, whose structure has been described as bcc. This phase has some characteristics similar to those of other high- $\mathrm{T}$ bcc phases of transition metals, for instance its dynamic instability at low temperatures. However, it also has some peculiarities, like an unusually high diffusion coefficient [17], which has been explined as due to an unusually low formation energy of self-interstitial atom defects [18]. This, and other peculiarities related to some alloying properties, led Starikov et al [19] to postulate that the $\gamma$ phase of $U$ is not really bcc, but rather quasi-bcc, meaning that for relatively long times (in the scale of a few ps) the average positions of the atoms do lie on the sites of a bcc lattice, but instantaneously the atomic positions resemble more those of a lattice where the central atom of the cube is displaced from the cube center towards one of the six faces, with the direction of the displacement randomly oriented in different cubes. This idea was in fact supported by ab-initio molecular dynamics simulations of the $\gamma$ phase, as well as by classical molecular dynamics simulations performed with a force-matched angular dependent potential (ADP) [18], specifically constructed to fit the properties of all solid phases and also some of
Table 1. Input data for the three thermodynamic states studied in this work. $\rho$ is the total ionic number density and $T$ is the temperature. $N_{c}$ is the total number of configurations.

\begin{tabular}{llll}
\hline system & $\rho\left(\AA^{-3}\right)$ & $T(\mathrm{~K})$ & $N_{c}$ \\
\hline \multirow{2}{*}{-U } & 0.043582 & 1455 & 18365 \\
& 0.043582 & 1705 & 27087 \\
& 0.043582 & 2050 & 13079 \\
\hline
\end{tabular}

the properties of the liquid. Notably, other types of effective potentials, in particular the EAM, lead to a bcc structure (not quasi-bcc) at the high temperatures corresponding to the $\gamma$ phase.

To our knowledge, there are only three theoretical studies of the properties of liquid $\mathrm{U}(\mathrm{l}-\mathrm{U})$. One of them was very comprehensive, but based on the use of an EAM effective potential specifically constructed for the liquid [20]. Another one was rather limited, since it provided just some information on the static structure and the diffusion coefficient of the liquid [21]. However, the interatomic forces were computed using first principles methods. Finally, a third study was the one by Starikov et al mentioned previously in relation to the solid $\gamma$ phase [19], as several atomic configurations corresponding to the liquid phase were used in the process of matching the ab initio forces to obtain the parameters of the ADP potential; however, no information was reported about the properties of the liquid.

We have performed a detailed analysis of the properties of liquid $\mathrm{U}$ at three temperatures and a constant density corresponding to the liquid's experimental one at the melting point. The forces are computed from first principles and the properties reported include several static structural functions, dynamic properties, and several thermophysical and transport properties. Similar to the case of the solid phase we find unique characteristics of liquid $U$ not shared, up to our knowledge, by any other single component liquid system.

\section{Computational method.}

We have performed FPMD simulations for liquid $\mathrm{U}$ at three temperatures and a constant density as shown in table 1. The FPMD simulations were performed with the DFT-based VASP code [22]. We have used the PBE generalized gradient (GGA) exchange-correlation functional [23] and the projector augmented wave (PAW) potentials for the electron-ion-core interaction [24] provided within the VASP distribution. We have taken 14 valence electrons (including $6 s, 6 p, 7 s, 5 f$ and $6 d$ electrons), and the plane-wave cutoff was set to 400 $\mathrm{eV}$, as recommended by the developers of the PAW potentials. Following previous studies $[15,16]$, we have 


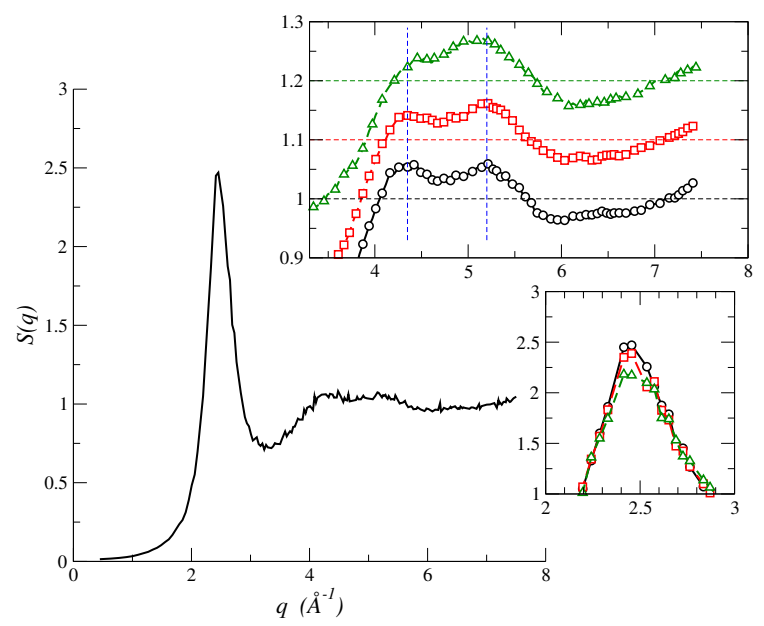

Figure 1. Structure factor, $S(q)$, of $1-\mathrm{U}$ at $1455 \mathrm{~K}$. The right inset shows the main peak region and the upper inset the second peak region for the three temperatures studied. Continuous black lines and circles correspond to $1455 \mathrm{~K}$, long-dashed red lines and squares to $1705 \mathrm{~K}$ and short-dashed green lines and triangles to $2050 \mathrm{~K}$. In the upper inset the results for the higher temperatures are shifted upwards for clarity.

not considered explicitly the SO interaction, which does not have a sizable influence on the interatomic forces, and therefore on the time evolution of the ionic configurations. The electronic iterations were considered converged when energy differences between iterations were within $10^{-6} \mathrm{eV}$.

We used cubic supercells with $120 \mathrm{U}$ atoms and periodic boundary conditions. For simulations of this size the single $\Gamma$ point is adequate enough for performing Brillouin-zone integrations for the computation of energies and forces. After initial equilibration, a number $N_{c}$ of equilibrium configurations (see table 1) in the NVT ensemble were used to compute the properties of the liquid. A time step of $3.5 \mathrm{fs}$ was used, so the total equilibrium simulation time spans from around 46 to around $95 \mathrm{ps}$, depending on the temperature.

For the sake of comparison, the only previous study of l-U using first principles methods by Hood et al [21] considered $54 \mathrm{U}$ atoms in the simulation cell, the total simulation time was around $3 \mathrm{ps}$, and a normconserving pseudopotential (that requires a much higher cutoff of $1360 \mathrm{eV}$ ) was used. Clearly our study will lead to a much smaller statistical uncertainties, and will allow the calculation of dynamic properties not available from these previous simulations. A final difference to comment is the density used for the study. Whereas we used the density of the liquid at the melting point, Hood et al held the density fixed to that of the $\alpha$ phase, so that their sample was liquid only above $2150 \mathrm{~K}$.

\section{Static structure and thermodynamic properties.}

The static structure factor, $S(q)$, of l-U obtained from our FPMD simulation at $1455 \mathrm{~K}$ is shown in figure 1. The main peak is located at $q=q_{p}=2.45$ $\AA^{-1}$, and its height and shape are quite typical among liquid systems. As the temperature increases (see right inset of figure 1) the position of the main peak does not change, while its height decreases as expected. The second peak of $S(q)$ shows a more peculiar behaviour, as can be observed in the upper inset of figure 1. It displays a clear double-peak shape for the three temperatures considered. The second subpeak is located at $5.20 \AA^{-1}$, and its height practically does not change with temperature. The low- $q$ side subpeak, however, gets more emphasized by decreasing temperature, while its position is basically the same, at $4.35 \AA^{-1}$, for the three temperatures. This is the first system so far where such a behaviour has been observed. The double-peak (or peak and shoulder) form of $S(q)$ in the region of its second maximum has been observed in quite a few recent measurements and simulations for liquid transition metals $[25,26,27,28]$, and also for liquid alkaline-earth metals [29, 30, 31]. However, in those systems it was the high- $q$ feature the one that got emphasized by decreasing temperatures, and this seemed to be correlated with the increase in the number of icosahedral local structures upon cooling. Whether the local structures in l-U are also of the same type or a different kind will be discussed below.

In the limit of long wavelengths the static structure factor is related to the isothermal compressibility of the liquid, $S(0)=\rho k_{B} T \chi_{T}$, or its inverse, the isothermal bulk modulus, $K_{T}=\chi_{T}^{-1}$. By extrapolating the FPMD $S(q)$ to long wavelengths we obtain bulk moduli of 78, 84 and $92 \mathrm{GPa}$ at 1455,1705 and $2050 \mathrm{~K}$ respectively. We are not aware of any experimental values to compare with. However there have been several estimates for $K_{T}$ at melting. One of them, from Tekuchev [32], differs largely from our result, being $34.4 \mathrm{GPa}$. Unfortunately, we have not been able to find out how this estimation was performed. This estimation of $K_{T}$ was one of the input data used by Belaschenko et al for the construction of the EAM potential that they used in their classical molecular dynamics study of l-U [20], and consequently it is not surprising that their resulting structural data are very different from ours, as we will comment below in relation to the pair correlation function, $g(r)$. A different estimation for $K_{T}$ of the liquid near melting was taken up by Fischer [33] by extrapolating values corresponding to the $\alpha$ phase at different temperatures. The final estimation was $62.4 \mathrm{GPa}$, which is very different from that of Tekuchev and in reasonable agreement with our re- 


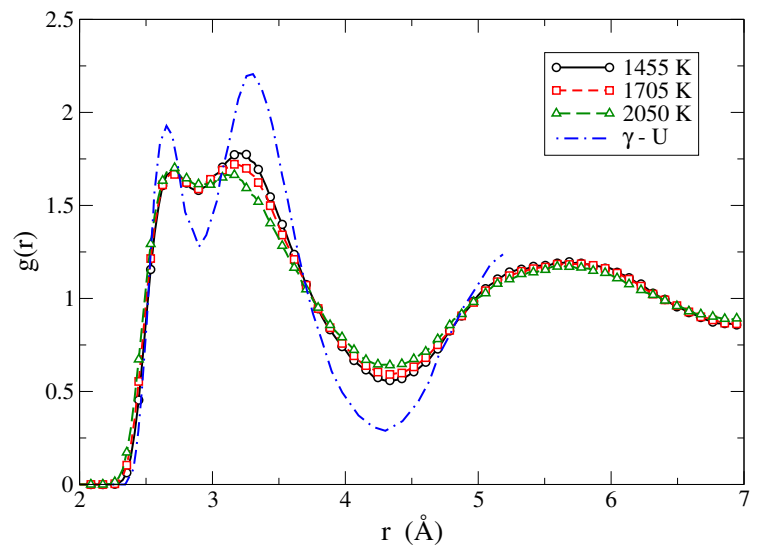

Figure 2. Pair correlation function, $g(r)$, of $\mathrm{l}-\mathrm{U}$ at the three temperatures considered. $\gamma$-U data are taken from [19]. Other lines and symbols have the same meaning as in figure 1 .

sult. We finally mention that an evaluation of the bulk modulus for the $\gamma$ phase from a fit of the experimental equation of state to a temperature independent model [34] yields a value of $113.3 \mathrm{GPa}$, so that our result at melting seems more reasonable than that of Tekuchev.

The pair correlation function, $g(r)$, is depicted in figure 2 for the three temperatures considered. The results of the FPMD calculations show very unusual characteristics for a one-component liquid. The height of the main peak of $g(r)$ is rather low, and its shape shows a very clear split structure. It is remarkable that this shape is quite similar to that obtained by Starikov et al in their FPMD study of the solid high temperature $\gamma$ phase, except for the smaller widths that correspond to the solid [19] because of the lower temperature. The positions of the maxima, however, are the same as the ones we obtained for the liquid.

The first shell of neighbours in the liquid, which spans up to $4.34 \AA$, is therefore divided into two subshells. The coordination number, as obtained from integration of the radial distribution function, $G(r)=4 \pi \rho r^{2} g(r)$, within the first shell is 14.2 for the three temperatures studied. It would be interesting to characterize the splitting of the neighbours within the first shell into the two subshells. That requires a way of separating the two subshells explicitly in a clearcut way. In order to perform this partition we have calculated the partial radial distribution functions, $G_{i}(r)$, that describe the distribution of distances from a central particle to its $i$-th neighbour. These functions verify that $G(r)=\sum_{i} G_{i}(r)$. It has been proposed [35] that the borderline between different shells in the liquid can be assigned to the $G_{i}(r)$ that displays a relative maximum in its width and a relative minimum in its height (note that all the $G_{i}(r)$ integrate to one). The $G_{i}(r)$ are easily calculated from the atomic positions during the simulation, and the results for $1455 \mathrm{~K}$ are

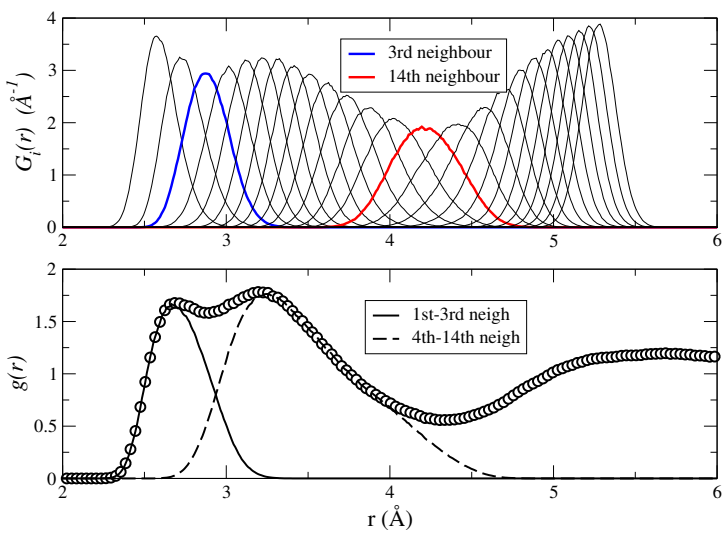

Figure 3. Upper panel: partial radial distribution functions, $G_{i}(r)$, of l-U at $1455 \mathrm{~K}$. The functions corresponding to the third and fourteenth neighbours are highlighted. Lower panel: partition of the first shell of $g(r)$ into a first subshell of three atoms and a second subshell of eleven atoms (from the fourth to the fourteenth neighbour).
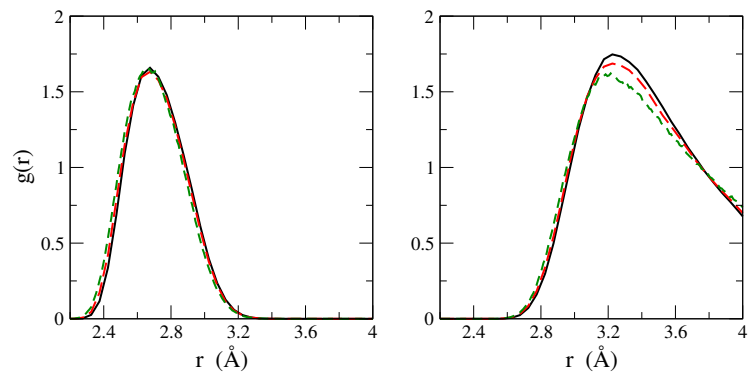

Figure 4. Decomposition of the first peak of $g(r)$ for the three temperatures studied. Left panel: first subshell. Right panel: second subshell. The meaning of the lines is the same as in figure 1.

shown in figure 3 . It shows that the conditions for separation of shells are met by $G_{3}(r)$ and $G_{14}(r)$, so that we can therefore describe the structure as having a subshell of 3 atoms within a first shell comprising 14 neighbours in total. The partition of the first peak of $g(r)$ into a first subshell of three closer neighbours and a second subshell of eleven neighbours, as shown also in figure 3, appears then quite natural. As a side note, the interpretation of Starikov et al of the $\gamma$ phase would lead to a first subshell with 4 atoms, rather than 3 as we find in the liquid. Unfortunately, no such analysis of the data was addressed in [19].

The results of performing such partition for the three temperatures considered are shown in figure 4. We see that indeed the first subshell is virtually independent of the temperature, having its maximum at $2.67 \AA$. A very slight displacement to smaller $r$ is however observed, which is compatible with the higher kinetic energy available to the particles at higher temperatures. The second subshell of the first peak 

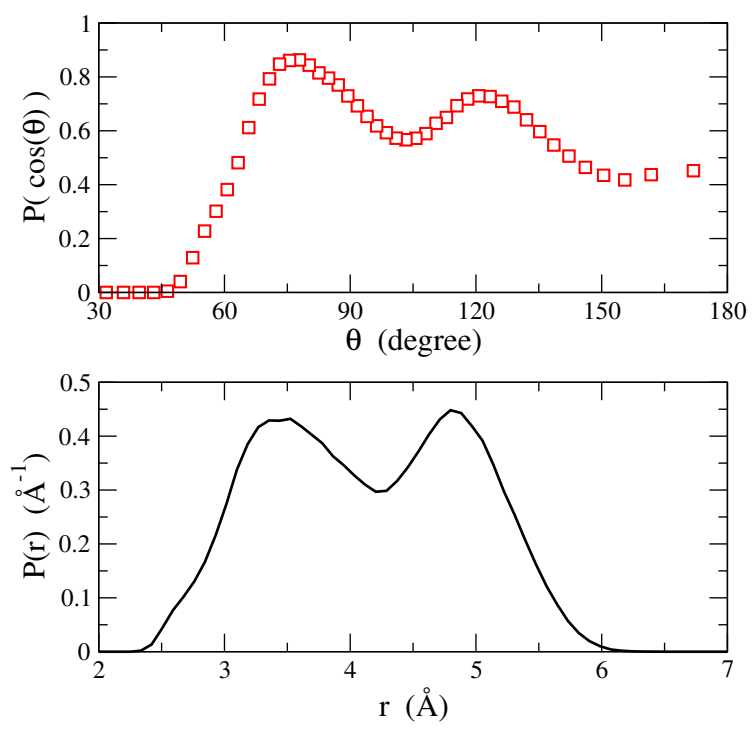

Figure 5. Distribution of angles (upper panel) and distances (lower panel) in the local pyramidal structure.

of $g(r)$ behaves however as expected for a system when the temperature rises, increasing the width and lowering the height of the maximum, whose location decreases from $3.24 \AA$ at $1455 \mathrm{~K}$ to $3.21 \AA$ at $2050 \mathrm{~K}$.

The lack of sensitivity of the first subshell of close neighbours to the temperature variations may suggest the possible existence of especially stable local structures, formed by the atom being considered and its first three neighbours. The four atoms obviously form a triangular pyramid, whose characteristics we describe below. We should mention that the quite standard procedure of using the common neighbour analysis to derive the type of local structure in the liquid is however not practical if we consider triangular pyramids. If we call the atom being considered as the vertex (atom number 0), and the three close neighbours the base of the pyramid (atoms 1, 2 and 3), we characterize the local structure by the angles $\theta_{102}, \theta_{103}$ and $\theta_{203}$, and by the distances among atoms of the base, $r_{12}, r_{13}$ and $r_{23}$. The distribution of angles and distances is shown in figure 5. Both are rather bimodal, with maxima for angles of 77 and 120 degrees, and for distances of 3.45 and $4.83 \AA$, with a very small shoulder near $2.67 \AA$, that corresponds to close neighbours. This shows that the atoms of the base are not in general close neighbours of each other, and therefore the pyramid is not especially compact, and is definitely not tetrahedron-like. On the contrary, the atoms of the base are either within the second subshell of the first coordination shell, or second neighbours, and the pyramids are distinctly flatter than tetrahedra.

It is worth noting that the previous studies of $1-\mathrm{U}$ $[20,21]$ did not show this type of structure, displaying $g(r)$ typical of simple liquids. In the case of the classical MD study by Belaschenko et al [20] we recall that they used EAM effective potentials, which are less accurate than DFT, but moreover they used the estimate of Tekuchev [32] for the liquid bulk modulus, which from our point of view is far too low, in order to construct their potential. Consequently, it is not surprising that the structural properties they obtained are substantially different from ours. The FPMD results of Hood et al [21] also showed a simple liquid like picture of the structure of $\mathrm{l}-\mathrm{U}$. The reasons underlying the differences with our data can be related to three aspects. First, the interaction between ions and valence electrons, which was taken into account with a normconserving pseudopotential vs PAW potentials in our study. The second one, possibly the most important, is the higher density considered in their study, which corresponded to the density of the solid $\alpha$ phase, vs the density of the liquid at melting that we have used. And third, related to this, the higher temperature required in their study so that the system was indeed liquid. In fact, we have performed a short simulation run (around 3 ps long) at the density and temperature of Hood's study but keeping the rest of parameters of our study, and the resulting $g(r)$, although still different from Hood's, shows a first coordintation shell which displays some asymmetry but does not indicate a clear splitting into two subshells.

\section{Collective dynamics and transport properties.}

We have investigated several collective dynamic properties including the intermediate scattering functions, dynamic structure factors, and longitudinal and transverse currents. These correlation functions have a dependence on the wavevector $\vec{q}$ which, for an isotropic system, reduces to a dependence on $q \equiv|\vec{q}|$ only.

\subsection{Definitions}

The microscopic number density and particle current in reciprocal space are defined as [36]

$\rho(\vec{q}, t)=\sum_{j=1}^{N} \exp \left[-i \vec{q} \cdot \vec{R}_{j}(t)\right]$,

and

$\vec{j}(\vec{q}, t)=\sum_{j=1}^{N} \vec{v}_{j}(t) \exp \left[-i \vec{q} \cdot \vec{R}_{j}(t)\right]$,

where $\vec{R}_{j}$ and $\vec{v}_{j}$ denote the position and velocity of particle $j$.

The microscopic current can be decomposed into its longitudinal and transverse components, parallel and perpendicular to the wavevector $\vec{q}$ respectively. 
The longitudinal term is completely determined by its module and can therefore be considered a scalar quantity, whereas the transverse current is a genuinely vectorial quantity.

$j_{L}(\vec{q}, t)=\frac{\vec{q}}{q} \cdot \vec{j}(\vec{q}, t)$

$\vec{j}_{T}(\vec{q}, t)=\vec{j}(\vec{q}, t)-\frac{\vec{q}}{q} j_{L}(\vec{q}, t)$

From these collective microscopic functions, the autocorrelation functions are defined as [36]

$F(q, t)=\frac{1}{N}\left\langle\rho(\vec{q}, t) \rho^{*}(\vec{q}, 0)\right\rangle$,

$C_{L}(q, t)=\frac{1}{N}\left\langle j_{L}(\vec{q}, t) j_{L}^{*}(\vec{q}, 0)\right\rangle$,

and

$C_{T}(q, t)=\frac{1}{2 N}\left\langle\vec{j}_{T}(\vec{q}, t) \cdot \vec{j}_{T}^{*}(\vec{q}, 0)\right\rangle$,

which are the intermediate scattering function, and the longitudinal and transverse current correlation functions. In the previous equations, the averages are taken over time origins and over wavevectors with the same module. Their Fourier Transforms into the frequency domain are the dynamic structure factor, $S(q, \omega)$, which describes the spectrum of collective density fluctuations, and the longitudinal and transverse current spectra, $C_{L}(q, \omega)$ and $C_{T}(q, \omega)$ respectively. Due to its definition, it is verified that $C_{L}(q, \omega)=\omega^{2} S(q, \omega) / q^{2}$, and consequently they contain the same information. However, the $\omega^{2}$ factor will tend to weaken the features for small frequencies and enhance those of higher frequencies.

These functions provide much information, since they not only describe the overall motion of the liquid's atoms, but also indicate the different mechanisms by which the system responds to perturbations of specific character, either relaxing them or propagating and attenuating them. Each of the relaxation modes contributes to the spectra with a function peaked at $\omega=0$, and each propagation mode contributes with a term peaked at the corresponding propagating frequency. The final form of the spectra is the sum of all contributions and therefore the spectra may or may not show peaks at non-zero frequencies, depending on the relative frequencies, widths and intensities of each mode, but the presence of such peaks give in general indication of existence of a propagating mode. When a peak appears in $S(q, \omega)$ we will denote the frequency of the peak as $\omega_{s}(q)$. Similarly we define $\omega_{L}(q)$ and $\omega_{T}(q)$ as the frequencies of the peaks in $C_{L}(q, \omega)$ and $C_{T}(q, \omega)$ when they exist. We additionally define several other characteristic frequencies, obtained from the second moments of the normalized spectra. This way we have [36]

$$
\begin{aligned}
& \omega_{0}^{2}(q)=\int d \omega \omega^{2} S(q, \omega) / F(q, t=0)=\frac{k_{B} T q^{2}}{m S(q)}, \\
& \omega_{2 \ell}^{2}(q)=\int d \omega \omega^{2} C_{L}(q, \omega) / C_{L}(q, t=0), \\
& \omega_{2 t}^{2}(q)=\int d \omega \omega^{2} C_{T}(q, \omega) / C_{T}(q, t=0) .
\end{aligned}
$$

These three characteristic frequencies are linear in $q$ in the hydrodynamic regime, i.e., for long wavelengths, $q \rightarrow 0$,

$$
\begin{aligned}
& \omega_{0}(q) \rightarrow c_{T} q, \\
& \omega_{2 \ell}(q) \rightarrow c_{\ell}^{\text {elast }} q, \\
& \omega_{2 t}(q) \rightarrow c_{t}^{\text {elast }} q,
\end{aligned}
$$

where $c_{T}$ is the isothermal sound velocity, and $c_{\ell}^{\text {elast }}$ and $c_{t}^{\text {elast }}$ are respectively the elastic (also called high-frequency or infinite-frequency) longitudinal and transverse velocities [36].

In this same regime, the frequencies of the maxima of $S(q, \omega)$ and $C_{L}(q, \omega)$ also vary linearly with $q$, with a common slope given by the adiabatic speed of sound, $c_{s}$, so that $\omega_{s}(q) \rightarrow c_{s} q$, and $\omega_{L}(q) \rightarrow c_{s} q$. Since a liquid cannot sustain shear waves on a macroscopic scale, $C_{T}(q, \omega)$ does not show side peaks for $q \rightarrow 0$, but after a propagation gap, $0<q<q_{g}$, viscoelasticity sets in and transverse waves start to propagate. In the vicinity of $q_{g}$, the maxima of the transverse current correlation function behave as [36]

$\omega_{T}(q) \rightarrow \alpha \sqrt{q^{2}-q_{g}^{2}}$.

All the velocities considered above can be related to the corresponding moduli, through the relations $K_{T}=\rho m c_{T}^{2}, \quad K_{s}=\rho m c_{s}^{2}, K_{\infty}=\rho m\left(c_{\ell}^{\text {elast }}\right)^{2}$, and $G_{\infty}=\rho m\left(c_{t}^{\text {elast }}\right)^{2}$, where $K_{T}$ is the isothermal bulk modulus, already introduced before, $K_{s}$ is the adiabatic bulk modulus, $K_{\infty}$ is the elastic or infinitefrequency bulk modulus and $G_{\infty}$ is the infinitefrequency shear or rigidity modulus. Note that $G_{\infty}$ should not be confused with the liquid's shear modulus, $G$, which is strictly zero since the liquid flows. Only if the liquid would be deeply undercooled near the glass transition temperature $G_{\infty}$ would be comparable to the corresponding $G$ of the (solid) glass [37], but this is not the case in the present study (and no glassy $\mathrm{U}$ has ever been synthesized).

Finally, we introduce two adimensional ratios. The first is the specific heats ratio, $\gamma=C_{P} / C_{v}$, which is also given by $\gamma=K_{s} / K_{T}$. The other one is the Poisson ratio, $\sigma=\left(3 K_{\infty}-2 G_{\infty}\right) /\left(6 K_{\infty}+2 G_{\infty}\right)$. Although for solids the Poisson ratio can be obtained from wave velocities, the absence of transverse waves for small $q$ in a liquid makes the previous definition more adequate. 


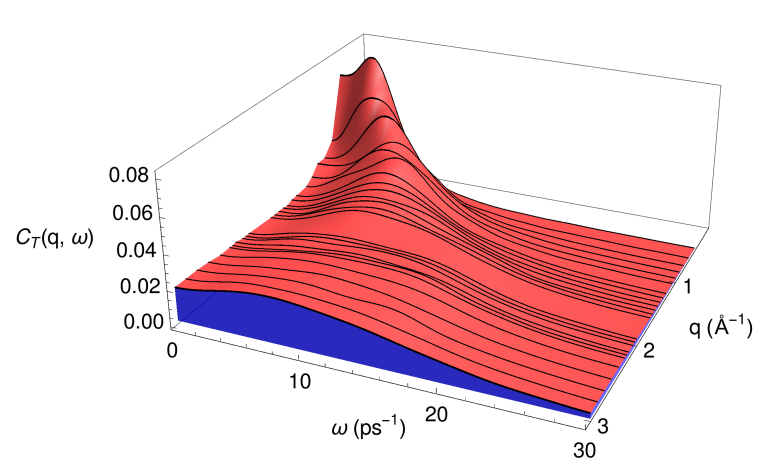

Figure 6. Normalized transverse current spectra, $C_{T}(q, \omega) / C_{T}(q, t=0)$, of l-U at $1455 \mathrm{~K}$.

\subsection{Transverse currents and viscosity}

We plot in figure 6 the FPMD normalized transverse current spectra obtained for $1455 \mathrm{~K}$. We observe that the smallest wavenumber compatible with our simulation setup, $q_{\min }=0.448 \AA^{-1}$, is already beyond the propagation gap for transverse waves, since the spectrum shows a well defined maximum. The analysis of the dispersion relation will be performed below. Here we just further mention the shape of $C_{T}(q, \omega)$ for $q$ approaching $2 \AA^{-1}$ and above. Even if only one maximum can be observed, the shape suggests the presence of two modes, with the high frequency one producing a shoulder rather than a peak. This type of behaviour has also been observed recently in a variety of liquid metals, and seen to become more pronounced at increased pressure, where the second mode does lead to a maximum in $C_{T}(q, \omega)[27,38,39]$. An explanation for the appearance of the second mode was put forward in the cases of 1-Zn and l-Sn [39] by using mode coupling ideas, where longitudinal and transverse modes couple indirectly within the whole wavevector range and not only at the same value of $q$. We also indicate that the higher temperature transverse currents behave in a qualitatively identical way.

A benefit of knowing the transverse current correlation functions is that it enables the calculation of the shear viscosity of the melt, which is a most important transport property that influences the way the liquid flows. The details of the procedure can be found in [36], but the basic idea is that the time integral of $C_{T}(q, t)$ can be used to define a wavevector dependent shear viscosity, $\eta(q)$, whose long wavelength limit is the macroscopic shear viscosity, $\eta$. To our knowledge there is only one reported measurement of the viscosities of l-U by Ofte [40], using an oscillating cup viscosimeter, in a temperature range from $1414 \mathrm{~K}$

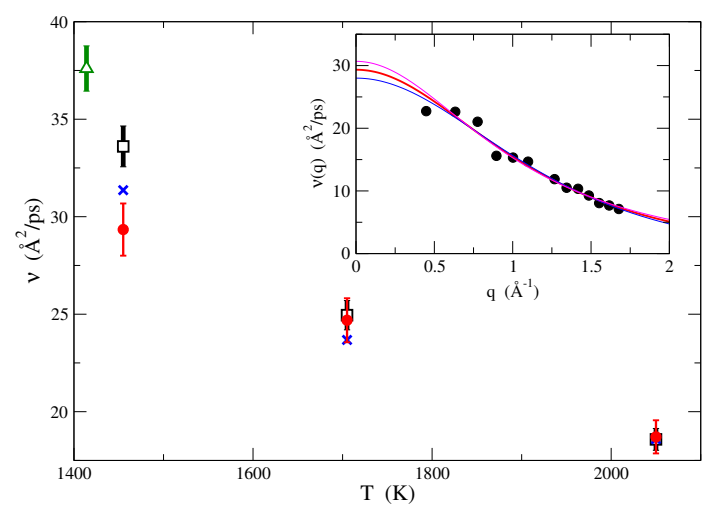

Figure 7. Kinematic viscosity of l-U. Experimental data for the dynamic viscosities have been converted to kinematic viscosities. Triangle: experimental measurement [40]. Squares: Ofte's correlations [40]. Crosses: IAEA correlation [3, 41]. Circles: present FPMD results. The inset shows the extrapolation procedure and a visual estimate of the uncertainty in the value of $\nu$.

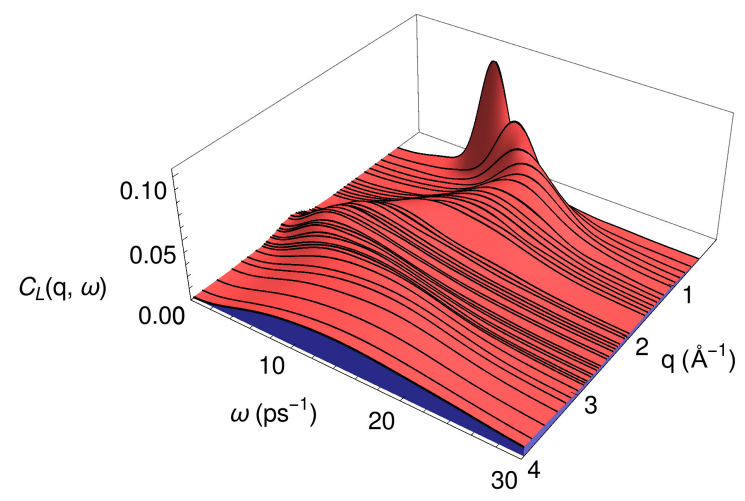

Figure 8. Normalized longitudinal current spectra, $C_{L}(q, \omega) / C_{L}(q, t=0)$, of l-U at $1455 \mathrm{~K}$.

(near the melting point) to $1521 \mathrm{~K}$. These data were then fitted with two different temperature dependent formulas. At the time of those measurements (1967) l$\mathrm{U}$ was found to be the most viscous pure liquid metal, with $\eta=6.5 \mathrm{cP}$ at the melting point. One reason for this high value is the large atomic mass. If we consider the kinematic viscosity, $\nu=\eta /(\rho m)$, then the values are similar to other liquid metals. There is an additional temperature dependent expression for the viscosity of l-U, which is the one recommended by the IAEA [3] up to $2973 \mathrm{~K}$, that surprisingly is not based on any actual measurements, but on a hard-sphere formula with parameters obtained from an estimation of the viscosity at melting through Andrade's formula [41].

We have calculated the kinematic viscosities using the procedure outlined above for the three temperatures studied, and the results are compared in figure 7 with the experimental meausurement at 

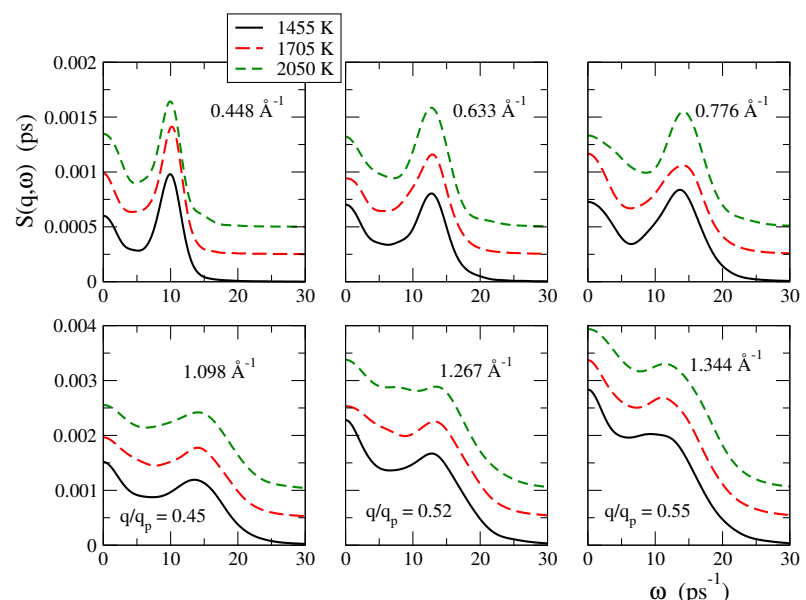

Figure 9. Dynamic structure factor, $S(q, \omega)$, of l-U at several $q$ values and different temperatures. The lines have the same meaning as in figure 1, and the results for higher temperatures have been shifted upwards for clarity.

melting, the two formulas provided by Ofte [40] (even if outside the proposed range of validity) and the one recommended by IAEA [3]. The overall comparison is satisfactory, but in any case it would be useful to have further measurements of $\eta$, even if only to update the IAEA recommendations. In fact, some containerless techniques have been developed for the measurements of the viscosity [42], which alleviate problems related to the reactivity of the sample at high temperatures, that may be suited for this type of system.

\subsection{Longitudinal currents}

The normalized longitudinal current spectra corresponding to $1455 \mathrm{~K}$ are shown in figure 8, where we can clearly appreciate the dispersion of the longitudinal mode as a function of wavevector. The results for the higher temperatures are qualitatively very similar. Below, we consider in more detail the dispersion relation $\omega_{L}(q)$ together with that corresponding to the dynamic structure factor, $\omega_{s}(q)$, and their second moments.

\subsection{Dynamic structure factor}

In figure 9 we plot the dynamic structure factors obtained from our simulations at the three temperatures considered, for several $q$ values below $q_{p}$ where clear side peaks, indicative of propagating collective density fluctuations, are observed. The corresponding frequencies are basically independent of the temperature.

As mentioned above, the presence of peaks (or shoulders) in $S(q, \omega)$ can be related to the existence of propagating modes. There have been recent discussions about the existence and nature of

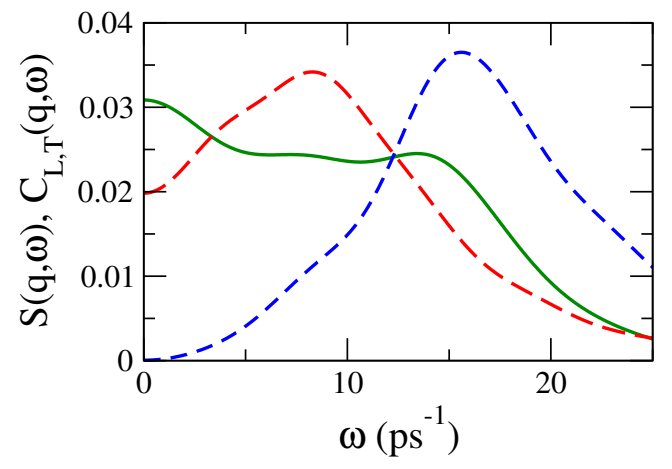

Figure 10. Dynamic structure factor and scaled (so as to fit in the graph) longitudinal and transverse current correlation spectra of $\mathrm{l}-\mathrm{U}$ at $2050 \mathrm{~K}$ for $q=0.52 q_{p}=1.627 \AA^{-1}$. Full green line is $S(q, \omega)$, long-dashed red line is $C_{T}(q, \omega)$ and short-dashed blue line is $C_{L}(q, \omega)$.

additional propagating modes in $S(q, \omega)$ in the region of wavevectors near $q_{p} / 2$, so called the first pseudoBrillouin zone. The first experimental observation of such feature was made by Hosokawa et al in an IXS measurement of the $S(q, \omega)$ in l-Ga [43]. Thereafter, similar excitation modes were detected in the $S(q, \omega)$ of l-Na, l-Sn, l-Fe, l-Cu and l-Zn [44, 45, 46, 47, 48]. Moreover, computer simulations have confirmed this type of feature for several liquids, adding $\mathrm{l}-\mathrm{Ni}$ to the list above $[43,49,50]$. The frequency of these excitations is numerically close to that of transverse waves at the same wavevector, and consequently these peaks were considered to be signatures of transverse acoustic modes visible in the $S(q, \omega)$ and incidentally also in $C_{L}(q, \omega)$. Their existence is quite clear, but this interpretation in terms of transverse excitations is not universally accepted because symmetry considerations inhibit a direct transverse-longitudinal coupling. Indirect coupling (mode coupling) may be the answer, but other possibilities about the nature of the excitations can also be put forward, and to date no clearcut explanation about their nature has been given. We see in figure 9 that $\mathrm{l}-\mathrm{U}$ also displays such an intermediate peak for $q=0.52 q_{p}$, but this only happens as the temperature is increased. In any case, the range of $q$ values where these modes are observed is very limited, since they are not visible in the neighbouring $q$-values allowed by the simulation setup. We also note again the coincidence in the frequencies of the additional feature in $S(q, \omega)$, that also appears in the longitudinal current spectrum, and the maximum in $C_{T}(q, \omega)$ as detailed in figure 10 .

\subsection{Dispersion relations and long wavelength limits}

Figures 11 and 12 show the dispersion relations associated to longitudinal and transverse excitations respectively. In the left panel of figure 11 we plot 

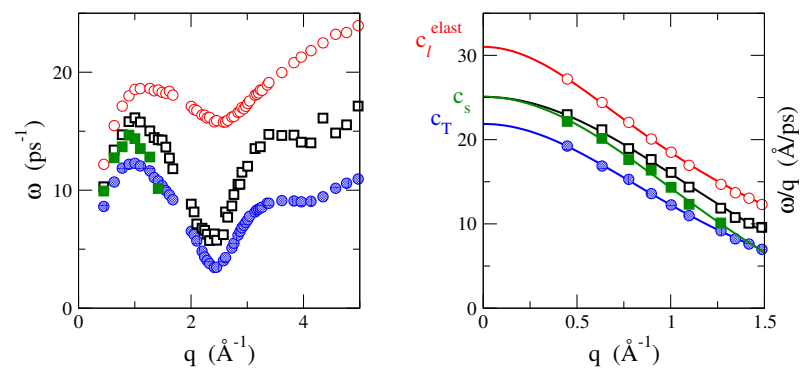

Figure 11. Left panel: longitudinal dispersion relations of l-U at $1450 \mathrm{~K}$. Right panel: corresponding phase velocities. Filled squares: $\omega_{s}(q)$, filled circles: $\omega_{0}(q)$, open squares: $\omega_{L}(q)$, open circles: $\omega_{2 \ell}(q)$.

the peaks and the second frequency moments of $S(q, \omega)$ and $C_{L}(q, \omega)$ at $1455 \mathrm{~K}$. As mentioned above $\omega_{s}(q)$ only exists in a limited $q$ range, but all the other characteristic longitudinal frequencies are visible at any q. In order to obtain estimates for the macroscopic velocities we have plotted in the right panel the phase velocities, $\omega(q) / q$, in a limited $q$ range, and extrapolated the data to $q \rightarrow 0$. Note that the data are compatible with the absence of any positive dispersion effect, and therefore we used a quadratic decreasing function of $q$ for performing the extrapolations. Additionally we have forced the same limit for the phase velocities associated to $\omega_{s}(q)$ and $\omega_{L}(q)$.

We have followed the same procedure for the second moments of the transverse current spectra, $\omega_{2 t}(q)$. In the case of the maxima we have fitted the $\omega_{T}(q)$ to (12). It turned out that the results for all temperatures could be well described by a single set of parameters (i.e. the propagation gap, $q_{g}$ and $\alpha$ could be considered temperature independent). Even though $\omega_{T}(q)$ behaves non-analytically, the variation not too close to $q_{g}$ is quasilinear and a transverse group velocity can be defined from the slope. Note that this is clearly smaller than the elastic transverse velocity, a behaviour similar to that of longitudinal waves, where the adiabatic sound velocity is smaller than the elastic longitudinal one.

From the obtained velocities we have computed the correspondig moduli, the specific heats ratio $\gamma$ and Poisson ratio $\sigma$. All the data for the three temperatures are compiled in table 2 . We observe that the adiabatic and elastic magnitudes are basically temperature independent. The isothermal magnitudes do change with temperature and also the value of $\gamma$ which depends on them. The value obtained for the Poisson ratio can be compared to those measured for policrystalline solids [51] at the $\alpha$ and $\beta$ phases, the first in the range 0.21 to 0.32 , the second around 0.5 , while no data was reported for the $\gamma$ phase.

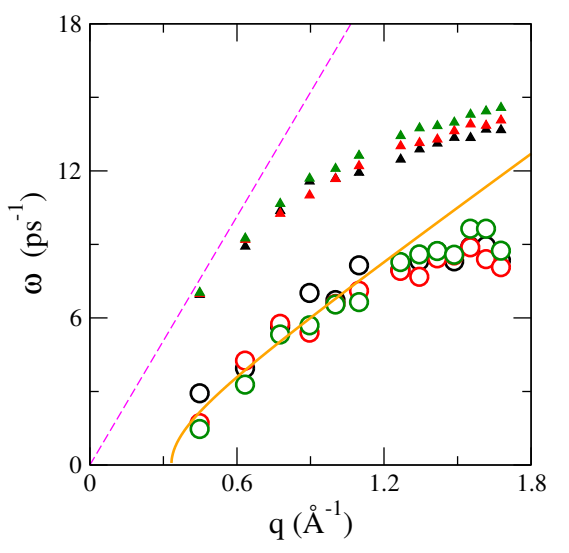

Figure 12. Dispersion relation of transverse waves for the three temperatures considered. Circles: $\omega_{T}(q)$, triangles: $\omega_{2 t}(q)$. Dashed line: linear dispersion with slope given by $c_{t}^{\text {elast }}$. Continuous line: common fit of the data corresponding to the three temperatures to (12). Color codes are the same as in figure 1.

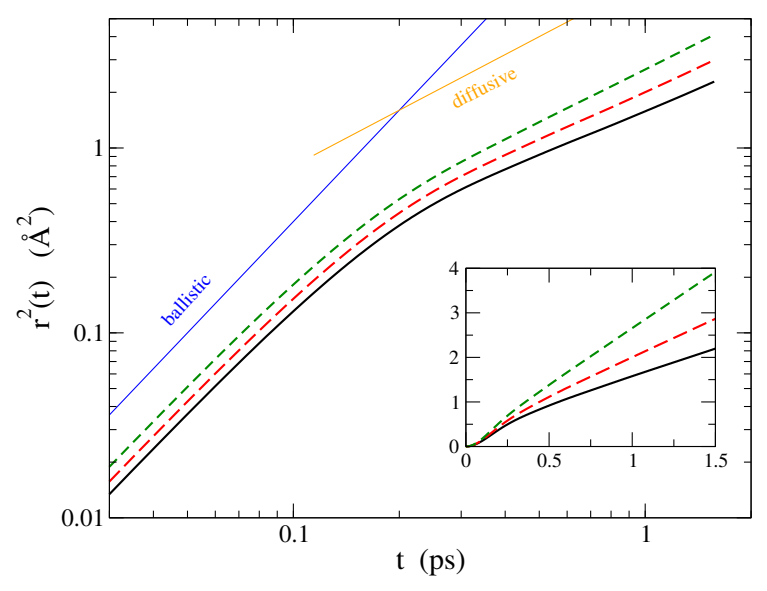

Figure 13. Mean square displacement of l-U for the three temperatures considered. Lines have the same meaning as in figure 1. The main figure is displayed with logarithmic axes, while the inset is shown with linear axes.

\section{Single particle dynamics and transport properties.}

The mean square displacement of an atom in the liquid, $\delta R^{2}(t)=\left\langle|\vec{R}(t)-\vec{R}(0)|^{2}\right\rangle$, and the normalized velocity autocorrelation function, $Z(t)=\langle\vec{v}(t) \cdot \vec{v}(0)\rangle /\left\langle v^{2}\right\rangle$, are two of the most important single-particle dynamic properties. For short times $\delta R^{2}(t)$ has a ballistic (quadratic in time) behaviour, since the atom does not feel the presence of others and the motion is dictated by the thermal velocity, $\sqrt{k_{B} T / m}$, whereas for long times it shows a diffusive (linear in time) behaviour, after undergoing many collisions. The diffusion coefficient, $D$, is in fact one sixth of its slope for long times. $Z(t)$ on the other hand, measures the way that the particle loses memory of its original velocity; it decays to zero 
Table 2. Isothermal sound velocity, adiabatic sound velocity, elastic longitudinal and transverse velocities, isothermal bulk modulus, adiabatic bulk modulus, infinite frequency bulk modulus and shear modulus, specific heats ratio and Poisson ratio for l-U at the three temperatures considered.

\begin{tabular}{lllllllllll}
\hline$T(\mathrm{~K})$ & $c_{T}(\mathrm{~m} / \mathrm{s})$ & $c_{s}(\mathrm{~m} / \mathrm{s})$ & $c_{\ell}^{\text {elast }}(\mathrm{m} / \mathrm{s})$ & $c_{t}^{\text {elast }}(\mathrm{m} / \mathrm{s})$ & $K_{T}(\mathrm{GPa})$ & $K_{s}(\mathrm{GPa})$ & $K_{\infty}(\mathrm{GPa})$ & $G_{\infty}(\mathrm{GPa})$ & $\gamma$ & $\sigma$ \\
\hline 1455 & 2190 & 2508 & 3101 & 1685 & 78 & 108 & 166 & 48.9 & 1.31 & 0.366 \\
1705 & 2150 & 2570 & 3098 & 1690 & 84 & 114 & 165 & 49.2 & 1.43 & 0.364 \\
2050 & 2120 & 2515 & 3199 & 1702 & 92 & 109 & 176 & 50.0 & 1.41 & 0.370 \\
\hline
\end{tabular}

for large times, since after many collisions the velocity becomes completely uncorrelated with the original one. At intermediate times it often takes negative values, related to the backscattering after a collision with particles in the near neighbours shell, and at short times its curvature is related to the Einstein frequency, $\omega_{E}$, which gives an idea about the frequency of the atomic vibrations inside the near neighbours cage [36]. Note that $D$ can also be obtained from the time integral of $Z(t)$.

The Fourier Transform of the velocity autocorrelation function, named the power spectrum, $Z(\omega)$, provides a more accurate description of the vibrating frequencies of the atoms, usually displaying a main maximum and either another maximum or a shoulder at a higher frequency, with $\omega_{E}$ lying somewhere between the two [36].

Figures 13 and 14 show respectively the mean square displacement and $Z(t)$ and its power spectrum. We observe the increase of the thermal velocity and also the diffusivity as the temperature is raised. The transition from the ballistic to the diffusive regime occurs basically in the same timescale for the three temperatures considered. The general shapes of $Z(t)$ and $Z(\omega)$ are rather usual, with the frequencies of the maximum and the shoulder insensitive to temperature but widened as the temperatue increases.

The observed emergence of the second trasverse mode for several liquid metals discussed previously has also been linked to the observation of a clear double peak structure in $Z(\omega)$. In particular, it has been proposed that the low/high frequency peak in $Z(\omega)$ is related to the low/high frequency peaks of $C_{T}(q, \omega)$ in the region where they hardly disperse, i.e. for $q$ somewhat above $q_{p} / 2$. There have been however several investigations about the origin of the two characteristic frequencies in the power spectrum, by resorting to the mode-coupling approximation of Gaskell and Miller [52], where the $Z(t)$ is evaluated in terms of two contributions representing the coupling of the single particle motion to the collective longitudinal and transverse currents respectively. The method has been used for several metallic systems such as l$\mathrm{Si}, \mathrm{l}-\mathrm{Na}, \mathrm{l}-\mathrm{Zn}$ and $\mathrm{l}-\mathrm{Sn}[27,39,49,53]$ and it has been shown that the low frequency peak and the high frequency shoulder/peak in the $Z(\omega)$ arise from

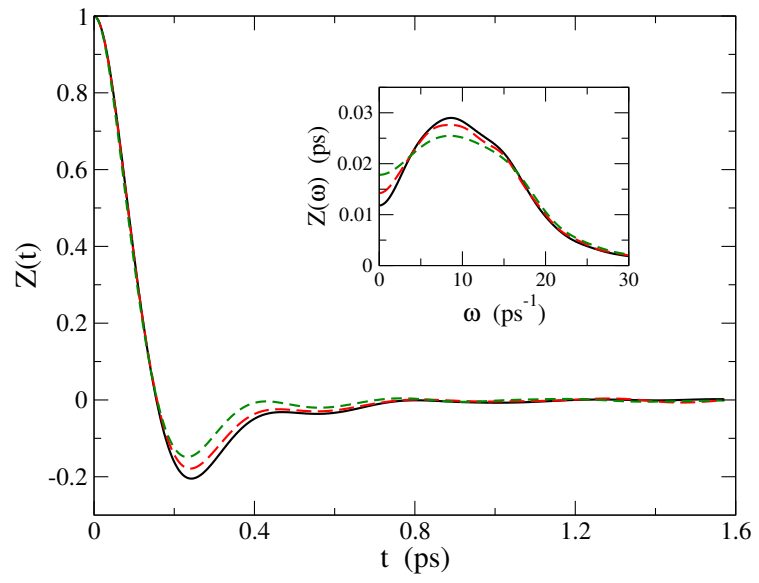

Figure 14. Normalized velocity autocorrelation function of $1-$ $\mathrm{U}$ at the three temperatures considered. The inset represents its corresponding power spectrum $Z(\omega)$. Lines have the same meaning as in figure 1.

Table 3. Calculated values of the self-diffusion coefficient $(D)$, Einstein frequency $\left(\omega_{E}\right)$, and kinematic shear viscosity $(\nu)$ for the different states. The uncertainties in $D$ reflect the differences between its calculation from the mean squared displacement and from the velocity autocorrelation funcion. Also shown are the ratios related to the fulfilment of the Stokes-Einstein relation.

\begin{tabular}{lllll}
\hline$T(\mathrm{~K})$ & $D\left(\frac{\AA^{2}}{\mathrm{ps}}\right)$ & $\omega_{E}\left(\mathrm{ps}^{-1}\right)$ & $\nu\left(\frac{\AA^{2}}{\mathrm{ps}}\right)$ & $\frac{D \nu}{T}\left(\frac{\AA^{4}}{\mathrm{ps}^{2} \mathrm{~K}}\right)$ \\
\hline 1455 & $0.21 \pm 0.01$ & 14.00 & $29.34 \pm 1.34$ & $4.15 \times 10^{-3}$ \\
1705 & $0.29 \pm 0.01$ & 14.25 & $24.69 \pm 1.13$ & $4.16 \times 10^{-3}$ \\
2050 & $0.42 \pm 0.01$ & 15.04 & $18.71 \pm 0.85$ & $3.83 \times 10^{-3}$ \\
\hline
\end{tabular}

the transverse and longitudinal components of $Z(t)$ respectively. Figure 15 suggests that this is also the case for l-U. The low frequency maximum of $Z(\omega)$ basically coincides with the frequencies of the main peaks of the transverse modes in the region above the first pseudo-Brillouin zone. On the other hand, the high frequency shoulder in $Z(\omega)$ basically coincides with the maximum in the longitudinal dispersion relation.

In the systems where the second transverse modes show up clearly it usually happens that its frequency above the first pseudo-Brillouin zone basically coincides with that of the maximum of the longitudinal dispersion relation. This was explained 


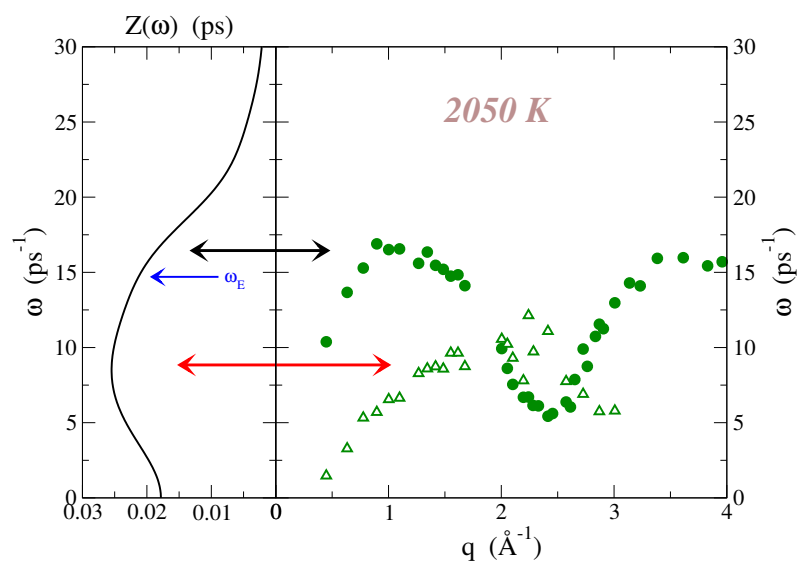

Figure 15. Relation between the power spectrum $Z(\omega)$ and the longitudinal and transverse dispersion relations for $\mathrm{l}-\mathrm{U}$ at 2050 K.

in [39] because in this $q$ region the weight of the coupling between transverse and longitudinal currents is maximum when the wavevector of $C_{L}(q, \omega)$ is around $q_{p} / 2$. As a consequence, the contribution of these high frequency transverse modes to $Z(\omega)$ adds up to the contribution of the longitudinal modes, which is always present.

The values of the diffusion coefficients and Einstein frequencies obtained from the FPDM simulations are given in table 3 . There is no experimental value of $D$ to compare with, but there is some estimation based on the measurement of the viscosity [40] and its relation with diffusivity through the Stokes-Einstein relation. Such procedure leads to a value of $D$ "exp" $=0.192$ $\AA^{2} /$ ps at the melting point.

The Stokes-Einstein relation connects the diffusion coefficient of a sphere of radius $r$ in a fluid with the fluid's shear viscosity. This relation can be written as $D \eta r / T=a$, where $a$ is a constant that only depends on the type of boundary conditions (slip or stick). It has however been applied in many cases to the motion of an atom (instead of a sphere) in a liquid, where $r$ is then taken as an effective atomic radius. In practical applications $r$ is often taken as the position of the maximum of $g(r)$. In the particular case of $\mathrm{l}-\mathrm{U}$ this is a troublesome criterion due to the shape of $g(r)$, but nevertheless, since the positions of the two subpeaks hardly change with temperature we can safely asume a temperature independent value for $r$. Also, taking into account that we have considered states with the same density, we can also substitute the dynamic viscosity by the kinematic one, which would lead to a simple change in the constant $a$. There is no clearcut reason for the applicability of the Stokes-Einstein relation for an atom in a liquid, and therefore having both values of $D$ and $\nu$ at several temperatures it is of interest to check if such relation holds. The values of $D \nu / T$ are shown in table 3 , where we observe that at the two lower temperatures the Stokes-Einstein relation holds accurately, but it does not at $2050 \mathrm{~K}$.

\section{Conclusions.}

We have found the structure of liquid $U$ as very peculiar among single component liquid metals. The static structure factor shows a shoulder on the low $q$ side of the second peak that gets enhanced upon cooling, in contrast with other liquid metals where it is the shoulder at the high $q$ side of the second peak that shows such behaviour. The real space structure is even more unusual, showing a split first peak of $g(r)$, with a temperature independent subshell of 3 close neighbours within a 14-atom first coordination shell. The arrangement of the atoms in the fisrt subshell is that of flattened triangular pyramids. The structure shows some resemblance of a recently proposed quasibcc structure for solid $\gamma$ uranium.

In the dynamic properties we find the appearance of additional propagating modes in $S(q, \omega)$ near the border of the first pseudo-Brillouin zone, but only at the highest temperature studied, that is, the state further apart from the solid. Except for this peculiarity the dynamic properties of liquid $U$ are not particularly different from those of simple liquid metals.

The evaluated elastic and adiabatic wave velocities and moduli change little with temperature, but one should keep in mind that these results have been obtained at constant density. Consequently we can infer that the values of these properties are mainly density driven.

Finally, we mention that there is in general a scarcity of experimental data for the liquid to compare with our results. This could possibly be caused by the challenging experimental conditions. However, some other related systems, as uranium dioxide, whose melting temperature is much higher and whose whose chemical reactivity at such high temperatures may also be problematic, has indeed been recently studied experimentally. Therefore, we expect that this study may spur some new measurements that could assess the predictions obtained in our simulations, and would be useful to reassess some recommendations for magnitudes such as the temperature dependent viscosity.

\section{Acknowledgments}

We acknlowledge the funding from Junta de Castilla y Leon (project VA124G18). LEG and DJG additionally acknowledge the support of the Spanish Ministry of Economy and Competitiveness (Project PGC2018093745-B-I00), which is also partially supported by 
FEDER funds.

\section{References}

[1] Fink J K 2000 J. Nucl. Mater. 2791

[2] Thermophysical Properties Database of Materials for Light Water Reactors and Heavy Water Reactors IAEATECDOC-1496, IAEA, Vienna (2006)

[3] Thermophysical Properties of Materials for Nuclear Engineering: A Tutorial and Collection of Data IAEA, Vienna (2009)

[4] http://www.gen-4.org/gif

[5] See, for instance, Baranov V G, Tenishev A V, Kuzmin R S, Pokrovskiy S A, Mikhalchik V V, Astafyev V A, Taubin M L and Solntseva E S 2016 Annals Nucl. Ener. 87784

[6] Lander G H, Fischer E S and Bader S D 1994 Adv. Phys. 431

[7] Fischer E S and McSkimin H J 1958 J. Appl. Phys. 291473 Le Bihan T, Heathman S, Idiri M, Lander G H, Wills J M, Lawson A C, and Lindbaum A 2003 Phys. Rev. B 67 134102

Crummett W P, Smith H G, Nicklow R M and Wakabayashi N 1979 Phys. Rev. B 196028

Manley M E, Fultz B, McQueeny R J, Brown C M, Hults W L, Smith J L, Thoma D J, Osborn R and Robertson J L 2001 Phys. Rev. Lett. 863076

Manley M E, Lander G H, Sinn H, Alatas A, Hults W L, McQueeney R J, Smith J L and Willit J 2003 Phys. Rev. B 67052302

Raymond S, Bouchet J, Lander G H, Le Tacon M, Garbarino G, Hoesch M, Rueff J-P, Krisch M, Lashley J C, Schulze R K and Albers R C 2011 Phys. Rev. Lett. 107136401

[8] Smirnova D E, Starikov S V and Stegailov V V 2012 J. Phys.: Condens. Matter 24015702

Pascuet M I, Bonny G and Fernández J R $2012 \mathrm{~J}$. Nucl. Mater. 424158

Beeler B, Deo C, Baskes M and Okuniewski M $2012 \mathrm{~J}$. Phys.: Condens. Matter 24075401

[9] Fernández J R and Pascuet M I 2014 Model. Simul. Mater. Sci. Eng. 22055019

[10] Li Y, Shan T-R, Liang T, Sinnot S B and Phillpot S R 2012 J. Phys.: Condens. Matter 24235403

Li Y, Chernatynskiy A, Kennedy J R, Sinnott S B and Phillpot S R 2016 J. Nucl. Mater. 4756

[11] Dai Y, Lu C H, Ren Q B, Lu L, Li J H and Liu B X 2012 J. Nucl. Mater. 427239

[12] Ladygin V V, Korotaev P Yu, A.V.Yanilkin A V and Shapeeva A V 2020 Comput. Mater. Sci. 172109333

[13] Hohenberg P and Kohn W 1964 Phys. Rev. 136 B864

Kohn W and Sham L J 1965 Phys. Rev. 140 A1133

[14] Soderlind P 2002 Phys. Rev. B 66085113

Soderlind P, Grabowski B, Yang L and Landa A 2012 Phys. Rev. B 85060301

[15] Crocombette J, Jollet F, Nga L and Petit T 2001 Phys. Rev. B 64104107

Taylor C 2008 Phys. Rev. B 77094119

Xiang S, Huang H and Hsiung L 2008 J. Nucl. Mater. 375 113

Shang S, Saengdeejing A, Mei Z, Kim D, Zhang H, Ganeshan S, Wang Y and Liu Z 2010 Comput. Mater. Sci. 48813

Adak S, Nakotte H, de Chatel P and Kiefer B 2011 Physica B 4063342

Li J H, Ren Q B, Lu C H, Dai Y and Liu B X 2012 J. Alloys Comp. 516139

Beeler B, Deo C, Baskes M and Okuniewski M 2013 J. Nucl. Mater. 433143

[16] Bouchet J 2008 Phys. Rev. B 77024113
[17] Adda Y and Kirianenko A 1959 J. Nucl. Mater. 1120

[18] Smirnova D E, Kuksin A Yu and Starikov S V 2015 J. Nucl. Mater. 458304

[19] Starikov S V, Kolotova L N, Kuksin A Yu, Smirnova D E and Tseplyaev V I 2018 J. Nucl. Mater. 499451

[20] Belaschenko D, Smirnova D E and Ostrovski O I 2010 High Temp. 48363

[21] Hood R Q, Yang L H and Moriarty J A 2008 Phy. Rev. B $\mathbf{7 8} 024116$

[22] Kresse G and Hafner J 1993 Phys. Rev. B 47558

Kresse G and Hafner J 1994 Phys. Rev. B 4914251

Kresse G and Furthmuller J 1996 Phys. Rev. B 5411169

Kresse G and Furthmuller J 1996 Comput. Mater. Sci. 6 15

[23] Perdew J P, Burke K and Ernzerhof M 1996 Phys. Rev. Lett. 773865

[24] Blochl P E 1994 Phys. Rev. B 5017953

Kresse G and Joubert D 1999 Phys. Rev. B 591758

[25] Schenk T, Holland-Moritz D, Simonet V, Bellissent R and Herlach D M 2002 Phys. Rev. Lett. 89075507

Kim T H and Kelton K F 2007 J. Chem. Phys. 126054513 Lee G W, Gangopadhyay A K, Hyers R W, Rathz T J, Rogers J R, Robinson D S, Goldman A I and Kelton K F 2008 Phys. Rev. B $\mathbf{7 7} 184102$

Inui M, Maruyama K, Kajihara Y and Nakada M 2009 Phys. Rev. B 80180201

[26] Jakse N and Pasturel A 2003 Phys. Rev. Lett. 91195501

Jakse N and Pasturel A 2004 J. Chem. Phys. 1206124

Jakse N, Le Bacq O and Pasturel A 2007 J. Non-Cryst. Solids 3533684

[27] Marqués M, González L E and González D J 2015 Phys. Rev. B 92134203

Marqués M, González L E and González D J 2016 J. Phys.: Condens. Matter 28075101

[28] del Rio B G, Rodríguez O, González L E and González D J 2017 Comput. Mat. Sci. 139243

[29] Tahara S, Fujii H, Yokota Y, Kawakita Y, Kohara S and Takeda S 2006 Physica B 385/386 219

[30] Şengül S, González D J and González L E 2009 J. Phys.: Condens. Matter 21115106

[31] del Rio B G and González L E 2014 J. Phys.: Condens. Matter 26465102

[32] Tekuchev V V 2005 Akusticheskoie issledovanie svoist elektronnych rasplavov (Volgograd: RPK Politekhnyk)

[33] Fischer E A 2000 Wissenschaftliche Berichte FZKA 6387

[34] Yoo C-S, Cynn H and Söderlind P 1998 Phys. Rev. B 57 10359

[35] McGreevy R L, Baranyai A and Ruff I 1986 Phys. Chem. Liq. 1647

[36] Balucani U and Zoppi M 1994 Dynamics of the Liquid State, (Oxford: Clarendon)

[37] Dyre J C 2006 Rev. Mod. Phys. 78953

[38] Bryk T, Ruocco G, Scopigno T and Seitsonen A P $2015 \mathrm{~J}$. Chem. Phys. 143110204

Bryk T, Demchuk T, Jakse N and Wax J F 2018 Frontiers in Physics 600006

Bryk T, Demchuk T and Jakse N 2019 Phys. Rev. B 99 014201

Jakse N and Bryk T 2019 J. Chem. Phys. 151034506

[39] del Rio B G and González L E 2017 Phys. Rev. B 95224201

del Rio B G, Chen M, González L E and Carter E A 2018 J. Chem. Phys. 149094504

[40] Ofte D 1967 J. Nucl. Mater. 2228

[41] Postovalov V G, Romanov E P, Kondrat'ev V P and Kononeko V I 2003 High Temp. 41762

[42] Langstaff D, Gunn M, Greaves G N, Marsing A and Kargl F 2013 Rev. Sci. Instr. 84124901

Ohisi Y, Kargl F, Nakamori F, Muta H, Kurosiki K and Yamanaka S 2017 J. Nucl. Mater. 487121

Kondo T, Muta H, Kurosaki K, Kargl F, Yamaji A, Furuya 
M and Ohisi Y 2019 Heliyon 5 e02049

[43] Hosokawa S, Inui M, Kajihara Y, Matsuda K, Ichitsubo T, Pilgrim W-C, Sinn H, González L E, González D J, Tsutsui S and Baron A Q R 2009 Phys. Rev. Lett. 102 105502

Hosokawa S, Inui M, Kajihara Y, Matsuda K, Ichitsubo T, Pilgrim W-C, Sinn H, González L E, González D J, Tsutsui S and Baron A Q R 2011 Eur. Phys. J. Special Topics 19685

[44] Giordano V M and Monaco G 2010 Proc. Nat. Acad. Sci. USA 10721985

[45] Hosokawa S, Munejiri S, Inui M, Kajihara Y, Pilgrim WC, Ohmasa Y, Tsutsui S, Baron A Q R, Shimojo F and Hoshino K 2013 J. Phys.: Condens. Matter 25112101

[46] Hosokawa S, Munejiri S, Inui M, Kajihara Y, Pilgrim W-C, Baron A Q R, Shimojo F and Hoshino K 2013 AIP Conf. Proc. 1518695

[47] Hosokawa S, Inui M, Kajihara Y, Tsutsui S and Baron A Q R 2015 J. Phys.: Condens. Matter 27194104

[48] Zanatta M, Sacchetti F, Guarini E, Orecchini A, Paciaroni A, Sani L and Petrillo C 2015 Phys. Rev. Lett. 114 187801

[49] Munejiri S, Shimojo F and Hoshino K 2012 Phys. Rev. B 86104202

[50] Marqués M, González D J and González L E 2016 Phys. Rev. B 94024204

del Rio B G, González L E and González D J 2017 J. Chem. Phys. 146034501

[51] Armstrong P E, Eash D T and Hockett J E 1972 J. Nucl. Mater. 45211

[52] Gaskell T and Miller S 1978 J. Phys. C 113749

Gaskell T and Miller S 1978 J. Phys. C 114839

Gaskell T and Miller S 1978 Phys. Lett. 66307

[53] Delisle A, González D J and Stott M J 2006 Phys. Rev. B 73064202

Delisle A, González D J and Stott M J 2006 J. Phys.: Condens. Matter 183591 